\title{
Trend and Outcomes of Direct Transcatheter Aortic Valve Replacement from a Single-Center Experience
}

\author{
Anthony A. Bavry · Seyed Hossein Aalaei-Andabili · Ki E. Park • \\ Calvin Y. Choi · Eddie W. Manning III · Wade W. Stinson
}

Received: June 22, 2018 / Published online: August 14, 2018

(C) The Author(s) 2018

\section{ABSTRACT}

Introduction: When transcatheter aortic valve replacement (TAVR) was introduced, pre-implantation balloon aortic valvuloplasty (BAV) was a routine part of the procedure. Smaller device profiles have resulted in selective use of BAV; however, there is a paucity of data about the trend in use of direct TAVR and the safety of this strategy.

Methods: All patients who underwent TAVR at a Veterans Affairs Medical Center from September 2013 to November 2016 were included in this retrospective analysis. We reviewed angiography films and verified with procedure reports to assess if direct TAVR was performed. Troponin $\mathrm{T}$ was assessed within $72 \mathrm{~h}$ after the

Enhanced digital features To view enhanced digital features for this article go to https://doi.org/10.6084/ m9.figshare.6930635.

A. A. Bavry $(\bowtie) \cdot$ S. H. Aalaei-Andabili ·

K. E. Park - C. Y. Choi

Department of Medicine, University of Florida, Gainesville, FL, USA

e-mail: anthony.bavry@va.gov

A. A. Bavry - K. E. Park - C. Y. Choi .

E. W. Manning III · W. W. Stinson

North Florida/South Georgia Veterans Health

System, Gainesville, FL, USA

E. W. Manning III · W. W. Stinson

Department of Surgery, University of Florida,

Gainesville, FL, USA
TAVR. Multivariate analysis examined the association between direct TAVR and periprocedural myocardial infarction (MI) or 1-year mortality.

Results: Overall, 207 patients were available for analysis. The mean follow-up was 13.3 months. A balloon-expandable valve was used $93.2 \%$ of the time, and $35.3 \%$ of patients were treated with conscious sedation. Periprocedural MI or 1 -year mortality occurred in $12.5 \%$ of the direct TAVR group versus $18.3 \%$ of the pre-implantation BAV group $(p=0.30)$. After controlling for potential confounding variables, direct TAVR was not associated with periprocedural MI or 1-year mortality.

Conclusions: Direct TAVR appears to be safe and is not associated with periprocedural MI or 1-year mortality. With current generation devices, this strategy can be considered for most patients undergoing TAVR.

Keywords: Aortic valve disease; Balloon angioplasty; Hemodynamic assessment; TAVR; Transcatheter valve implantation

\section{INTRODUCTION}

Avoiding pre-implantation balloon aortic valvuloplasty (BAV) during transcatheter aortic valve replacement (TAVR) is termed direct TAVR and is a feasible strategy. The United Kingdom registry found no increase in adverse 
events from this approach; however, direct TAVR was only used in the minority of patients [1]. Accordingly, we sought to examine the trend and association between direct TAVR and periprocedural myocardial infarction or 1-year mortality in a Veterans Affairs Hospital where this strategy is now common and periprocedural cardiac enzymes are routinely measured.

\section{METHODS}

This study was approved by our Institutional Review Board. This article is based on previously conducted studies and does not involve any new studies of human participants or animal subjects performed by any of the authors. TAVR procedures were performed between September 2013 and November 2016. Direct TAVR was performed according to operator discretion. Postoperative troponin $\mathrm{T}$ values and transthoracic echocardiography were obtained on all patients. Periprocedural myocardial infarction was defined according to the Valve Academic Research Consortium-2 (troponin $\mathrm{T}>15 \times$ upper limit of normal) [2]. Multivariate logistic regression examined independent predictors for perioperative myocardial infarction or 1-year mortality. Statistics were performed with SPSS software (Version 22, IBM Co., Armonk, NY, USA).

\section{RESULTS}

Overall, 207 patients were available for analysis. The median follow-up was 13.3 months (mean, $15.3 \pm 11.6$ months). The frequency of direct TAVR increased over time $[14 \%$ in the first quarter of the study period, $30 \%$ in the second quarter of the study period, $80 \%$ in the third quarter of the study period, and $83 \%$ in fourth quarter of the study period $(p<0.001)]$. Among the total cohort, the mean age was $77.6 \pm 8.7$ years; mean left-ventricular ejection fraction; $52.3 \pm 10.4 \%$; and mean stroke volume index, $38.2 \pm 9.9 \mathrm{cc} / \mathrm{m}^{2}$. Table 1 provides complete study characteristics. A balloon-expandable valve was used in $93.2 \%(n=193)$ and conscious sedation in $35.3 \%(n=73)$. In the direct TAVR group versus the pre-implantation BAV group, there was a higher frequency of conscious sedation, trans-femoral access, and second/third-generation valves $(p<0.05$ for each).

The mean troponin $T$ value was $0.093 \pm 0.2 \mathrm{ng} / \mathrm{ml}$ in the direct TAVR group and $0.13 \pm 0.18 \mathrm{ng} / \mathrm{ml}$ in the pre-implantation BAV group $(p=0.16)$. Periprocedural myocardial infarction or 1-year mortality occurred in $12.5 \%$ of the direct TAVR group versus $18.3 \%$ of the pre-implantation BAV group $(p=0.30)$ (Table 2). Heart rate-adjusted diastolic delta (HR-DD) $<25$ after valve implantation occurred in $9.1 \%$ of the direct TAVR group (5/55) compared with $2.4 \%$ of the pre-implantation BAV group $(3 / 125)(p=0.06)$ [3]. Univariate predictors of periprocedural myocardial infarction or 1-year mortality included history of coronary artery bypass grafting, mean pulmonary pressure $\geq 45 \mathrm{~mm} \mathrm{Hg}$, general anesthesia, transapical access, TAVR in the first half of the program, and HR-DD $<25$. Direct TAVR was not associated with periprocedural myocardial infarction or 1-year mortality with multivariate analysis after controlling for history of coronary artery bypass grafting, mean pulmonary pressure $\geq 45 \mathrm{~mm} \mathrm{Hg}$, and HR-DD $<25$. The results were the same in a subgroup analysis that was restricted to transfemoral access $(n=170)$. Table 3 provides complete procedural outcomes.

\section{DISCUSSION}

In this retrospective analysis, the proportion of veterans undergoing direct TAVR increased significantly over time, reaching $83 \%$ in the last quarter of the study period. While there was a numerical difference favoring a lower incidence of periprocedural myocardial infarction or 1-year mortality with direct TAVR, this relationship was not significant after controlling for multiple confounding variables (general anesthesia, stroke volume index $<35$, mean pulmonary pressure $\geq 45 \mathrm{~mm} \mathrm{Hg}$, HR-DD $<25$ [3], and aortic insufficiency $\geq$ moderate).

When TAVR was first introduced, pre-implantation BAV was considered a necessary part 
Table 1 Study characteristics of the cohort

\begin{tabular}{|c|c|c|c|c|}
\hline Baseline characteristic & $\begin{array}{l}\text { Total } \\
(n=207)\end{array}$ & $\begin{array}{l}\text { Direct TAVR } \\
(n=136)\end{array}$ & $\begin{array}{l}\text { Pre-implantation } \\
\text { BAV }(n=71)\end{array}$ & $P$ value \\
\hline Age, mean years $\pm S D$ & $77.6 \pm 8.7$ & $76.8 \pm .5$ & $78.9 \pm 7.5$ & 0.08 \\
\hline Body mass index, mean $\mathrm{kg} / \mathrm{m}^{2} \pm \mathrm{SD}$ & $28.7 \pm 5.7$ & $29.1 \pm 5.5$ & $27.8 \pm 6.2$ & 0.12 \\
\hline Diabetes mellitus, $n$ & $103(49.7 \%)$ & $67(49.3 \%)$ & $36(50.7 \%)$ & 0.76 \\
\hline Peripheral vascular disease, $n$ & $93(44.9 \%)$ & $67(49.3 \%)$ & $26(36.6 \%)$ & 0.10 \\
\hline Coronary artery bypass grafting, $n$ & $77(37.2 \%)$ & $52(37.4 \%)$ & $25(35.2 \%)$ & 0.87 \\
\hline Percutaneous coronary intervention, $n$ & $58(28.0 \%)$ & $38(27.9 \%)$ & $20(28.2 \%)$ & 1.0 \\
\hline Stroke/transient ischemic attack, $n$ & $32(15.4 \%)$ & $20(14.7 \%)$ & $12(16.9 \%)$ & 0.70 \\
\hline Atrial fibrillation, $n$ & $65(31.4 \%)$ & $45(33.1 \%)$ & $20(28.2 \%)$ & 0.53 \\
\hline Chronic obstructive pulmonary disease, $n$ & $77(37.2 \%)$ & $50(36.7 \%)$ & $27(38.0 \%)$ & 0.88 \\
\hline Creatinine, mean $\mathrm{mg} / \mathrm{dl} \pm \mathrm{SD}$ & $1.29 \pm 0.85$ & $1.30 \pm 0.8$ & $1.28 \pm 0.4$ & 0.89 \\
\hline \multicolumn{5}{|l|}{ Pre-operative echo } \\
\hline LVEF, mean $\mathrm{mm} \mathrm{Hg} \pm \mathrm{SD}$ & $52.3 \pm 10.4$ & $52.3 \pm 10.9$ & $52.4 \pm 9.4$ & 0.94 \\
\hline Mean aortic valve gradient, mean $\mathrm{mm} \mathrm{Hg} \pm \mathrm{SD}$ & $40.7 \pm 12.2$ & $39.1 \pm 10.9$ & $43.6 \pm 14.0$ & 0.01 \\
\hline Mean gradient $<40 \mathrm{~mm} \mathrm{Hg}$, $n$ & $95(45.9 \%)$ & $67(51.6 \%)$ & $28(40.9 \%)$ & 0.21 \\
\hline \multicolumn{5}{|l|}{ Pre-operative cath } \\
\hline$S V_{i}^{*}$, mean $\mathrm{cc} / \mathrm{m}^{2} \pm \mathrm{SD}$ & $36.9 \pm 9.5$ & $37.6 \pm 9.8$ & $35.8 \pm 8.7$ & 0.18 \\
\hline $\mathrm{SVi}<35 \mathrm{cc} / \mathrm{m}^{2}, n$ & $91 / 205(44.9 \%)$ & $58 / 135(42.9 \%)$ & $33 / 70(47.1 \%)$ & 0.65 \\
\hline Pulmonary pressure, mean $\mathrm{mm} \mathrm{Hg} \pm \mathrm{SD}$ & $27.9 \pm 10.2$ & $27.5 \pm 9.8$ & $28.8 \pm 10.9$ & 0.47 \\
\hline Pulmonary pressure $(\text { mean } \geq 45 \mathrm{~mm} \mathrm{Hg})^{* *}, n$ & $10 / 153(6.5 \%)$ & $6 / 101(5.9 \%)$ & $4 / 52(7.7 \%)$ & 0.73 \\
\hline \multicolumn{5}{|l|}{ Pre-operative CT } \\
\hline Valve area oversize $\geq 15 \%^{* * *}, n$ & $63 / 179(35.2 \%)$ & $38 / 114(33.3 \%)$ & $32 / 65(49.2 \%)$ & 0.001 \\
\hline \multicolumn{5}{|l|}{ Procedural details } \\
\hline General anesthesia, $n$ & $134(64.7 \%)$ & $72(52.9 \%)$ & $62(87.3 \%)$ & $<0.001$ \\
\hline Conscious sedation, $n$ & $73(35.3 \%)$ & $64(47.1 \%)$ & $9(12.7 \%)$ & $<0.001$ \\
\hline Native implant, $n$ & $191(92.3 \%)$ & $124(91.2 \%)$ & $67(80.3 \%)$ & 0.58 \\
\hline Valve-in-valve implant, $n$ & $16(7.7 \%)$ & $12(8.8 \%)$ & $4(6.6 \%)$ & 0.58 \\
\hline Trans-apical access, $n$ & $21(10.1 \%)$ & $12(8.8 \%)$ & $9(12.7 \%)$ & 0.63 \\
\hline Trans-femoral access, $n$ & $170(82.1 \%)$ & $109(77.9 \%)$ & $61(85.9 \%)$ & 0.63 \\
\hline Balloon expandable valve, $n$ & $193(93.2 \%)$ & $125(91.9 \%)$ & $68(95.8 \%)$ & 0.55 \\
\hline Sapien, $n$ & $22(10.6 \%)$ & $6(4.4 \%)$ & $16(22.5 \%)$ & $<0.001$ \\
\hline Sapien XT, $n$ & $52(25.1 \%)$ & $11(8.1 \%)$ & $41(57.7 \%)$ & $<0.001$ \\
\hline
\end{tabular}


Table 1 continued

\begin{tabular}{|c|c|c|c|c|}
\hline Baseline characteristic & $\begin{array}{l}\text { Total } \\
(n=207)\end{array}$ & $\begin{array}{l}\text { Direct TAVR } \\
(n=136)\end{array}$ & $\begin{array}{l}\text { Pre-implantation } \\
\text { BAV }(n=71)\end{array}$ & $\overline{P \text { value }}$ \\
\hline Sapien S3, $n$ & $119(57.5 \%)$ & $108(79.4 \%)$ & $11(15.5 \%)$ & $<0.001$ \\
\hline Self expandable valve, $n$ & $14(6.8 \%)$ & $11(8.1 \%)$ & $3(4.2 \%)$ & 0.55 \\
\hline CoreValve, $n$ & $9(4.3 \%)$ & $8(5.9 \%)$ & $1(1.4 \%)$ & 0.2 \\
\hline Evolut R, $n$ & $5(2.4 \%)$ & $3(2.2 \%)$ & $2(2.8 \%)$ & 1.0 \\
\hline Evolut Pro & $0(0 \%)$ & $0(0 \%)$ & $0(0 \%)$ & 1.00 \\
\hline Total pacing runs $\geq 2$ & $130(62.8 \%)$ & $60(44.1 \%)$ & $70(98.6 \%)$ & $<0.001$ \\
\hline Contrast, mean $\mathrm{cc} \pm \mathrm{SD}$ & $128.9 \pm 51.4$ & $128.9 \pm 55.7$ & $128.7 \pm 42.4$ & 0.53 \\
\hline
\end{tabular}

$B A V$ indicates balloon aortic valvuloplasty, $C T$ indicates computed tomography, $L V E F$ indicates left ventricular ejection fraction, $S D$ indicates standard deviation, $S V i$ indicates stroke volume index

*Preferentially obtained from pre-operative catheterization $(n=146)$; however, echocardiogram-derived SVi used if the former not available $(n=59)$

** Obtained from pre-operative catheterization $(n=153)$

${ }^{* * *}$ Applies to balloon expandable valve implanted in a native aortic valve

Table 2 Frequencies of troponin $\mathrm{T}$ elevation after transcatheter aortic valve replacement (TAVR)

\begin{tabular}{lllll}
\hline & $\begin{array}{l}\text { Total cohort } \\
(\boldsymbol{n}=\mathbf{1 8 9})\end{array}$ & $\begin{array}{l}\text { Direct TAVR } \\
(\boldsymbol{n}=\mathbf{1 2 8})\end{array}$ & $\begin{array}{l}\text { Pre-implantation BAV } \\
(\boldsymbol{n}=\mathbf{6 1})\end{array}$ & P value \\
\hline Troponin T mean ng/ml $\pm \mathrm{SD}$ & $0.10 \pm 0.2$ & $0.093 \pm 0.20$ & $0.13 \pm 0.18$ & 0.16 \\
$\geq 0.45(>15 \times \mathrm{ULN}), n(\%)$ & $5(2.6 \%)$ & $2(1.6 \%)$ & $3(4.9 \%)$ & 0.33 \\
$\geq 0.3(>10 \times \mathrm{ULN}), n(\%)$ & $9(4.8 \%)$ & $4(3.1 \%)$ & $5(8.2 \%)$ & 0.15 \\
$\geq 0.15(>5 \times \mathrm{ULN}), n(\%)$ & $41(21.7 \%)$ & $23(18.0 \%)$ & $18(29.5 \%)$ & 0.09 \\
$\geq 0.09(>3 \times \mathrm{ULN}), n(\%)$ & $71(37.6)$ & $42(32.8 \%)$ & $29(47.5 \%)$ & 0.06 \\
$\geq 0.03(>\mathrm{ULN}), n(\%)$ & $138(73.0 \%)$ & $87(68.0 \%)$ & $51(83.6 \%)$ & 0.02 \\
& $(n=207)$ & $(n=136)$ & $(n=71)$ & 0.66 \\
30 -day mortality, $n(\%)$ & $6(2.9 \%)$ & $5(3.7 \%)$ & $1(1.4)$ & 0.52 \\
$\begin{array}{l}\text { 1-year mortality, } n \text { (\%) } \\
\text { Periprocedural MI or } 1 \text {-year }\end{array} \quad 27(13.0 \%)$ & $16(11.8 \%)$ & $11(15.5 \%)$ & 0.30 \\
mortality, $n$ (\%) & $30(14.5 \%)$ & $17(12.5 \%)$ & $13(18.3 \%)$ & \\
\hline
\end{tabular}

$B A V$ indicates balloon aortic valvuloplasty, $M I$ indicates myocardial infarction, $U L N$ indicates upper limit of normal

of the procedure. This was done to avoid crossing failures or hypotension that could potentially result from the bulky TAVR valve occupying a tight orifice [4]. With the introduction of the self-expandable valve and later- generation balloon-expandable valves, experience accumulated that avoiding BAV is feasible and can streamline the procedure [1]. Accordingly, this strategy appears to be increasing in popularity worldwide. 
Table 3 Frequencies of post transcatheter aortic valve replacement (TAVR) complications

\begin{tabular}{|c|c|c|c|c|}
\hline & $\begin{array}{l}\text { Total cohort } \\
(n=207)\end{array}$ & $\begin{array}{l}\text { Direct TAVR } \\
(n=136)\end{array}$ & $\begin{array}{l}\text { Pre-implantation BAV } \\
(n=71)\end{array}$ & $P$ value \\
\hline $\begin{array}{l}\text { Immediate procedure success, } \\
n(\%)\end{array}$ & $202(97.6 \%)$ & $132(97.1 \%)$ & $70(98.6 \%)$ & 0.66 \\
\hline 30-day mortality, $n$ (\%) & $6(2.9 \%)$ & $5(3.7 \%)$ & $1(1.4 \%)$ & 0.66 \\
\hline Cardiac mortality, $n(\%)$ & $3(1.4 \%)$ & $3(2.2 \%)$ & $0(0 \%)$ & 0.55 \\
\hline Stroke, $n(\%)$ & $2(1 \%)$ & $2(1.5 \%)$ & $0(0 \%)$ & 0.54 \\
\hline Major bleeding, $n(\%)$ & $2(1.0 \%)$ & $1(0.7 \%)$ & $1(1.4 \%)$ & 1.0 \\
\hline $\begin{array}{l}\text { Major vascular complication, } \\
n(\%)\end{array}$ & $4(1.9 \%)$ & $1(0.7 \%)$ & $3(4.2 \%)$ & 0.12 \\
\hline $\begin{array}{l}\text { Coronary artery obstruction, } \\
n(\%)\end{array}$ & $0(0 \%)$ & $0(0 \%)$ & $0(0 \%)$ & 1.0 \\
\hline $\begin{aligned} \geq & \text { Moderate paravalvular AI, } \\
& n(\%)\end{aligned}$ & $3(1.4 \%)$ & $0(0 \%)$ & $3(4.2 \%)$ & 0.04 \\
\hline Mild paravalvular AI, $n$ (\%) & $24(11.6 \%)$ & $13(9.6 \%)$ & $11(15.5 \%)$ & 0.25 \\
\hline
\end{tabular}

$A I$ aortic insufficiency, $B A V$ balloon aortic valvuloplasty

It is interesting that approximately threefourths of patients had detectable cardiac enzyme elevation ( $>$ upper limit of normal); however, this does not appear to be clinically relevant [2]. Another study found that the median troponin value was lower in the direct TAVR group compared with the pre-implantation BAV group; however, that study did not assess for VARC-2 periprocedural myocardial infarction [5]. While direct TAVR is likely preferential with current-generation devices, selective BAV still has a role in certain cases. In our own institution, we perform selective BAV with aortic valve area $\leq 0.6 \mathrm{~cm}^{2}$ or mean trans-aortic gradient $\geq 60 \mathrm{~mm} \mathrm{Hg}$, bicuspid aortic valve, horizontal aorta, and during simultaneous aortography when there is concern about coronary artery occlusion. A limitation of the present study was single-center design conducted almost exclusively in men. A strength of the study is incorporation of important hemodynamic variables such as stroke volume index, which are frequently lacking in other studies, especially large registries. Additional information on this topic will come from the randomized DIRECTAVI trial, which is designed to evaluate the non-inferiority of direct TAVR compared with pre-implantation BAV [6].

\section{CONCLUSIONS}

In summary, in a single-center experience, direct TAVR increased significantly over time and was not associated with periprocedural myocardial infarction or 1-year mortality. With current generation devices, this strategy can be considered for most patients undergoing TAVR.

\section{ACKNOWLEDGEMENTS}

Funding. No funding or sponsorship was received for this study or publication of this article.

Authorship. All named authors meet the International Committee of Medical Journal Editors (ICMJE) criteria for authorship for this article, take responsibility for the integrity of 
the work as a whole, and have given their approval for this version to be published.

Disclosures. Dr. Anthony A. Bavry discloses the following relationship-Honorarium from American College of Cardiology and Advisory Board for Cardiovascular Systems, Inc. Seyed Hossein Aalaei-Andabilia, Ki E. Park, Calvin Y. Choi, Eddie W Manning III, and Wade W. Stinson have nothing to disclose.

Compliance with Ethics Guidelines. This article is based on previously conducted studies and does not involve any new studies of human or animal subjects performed by any of the authors.

Data Availability. The datasets generated during and/or analyzed during the current study are available from the corresponding author on reasonable request.

Open Access. This article is distributed under the terms of the Creative Commons Attribution-NonCommercial 4.0 International License (http://creativecommons.org/licenses/ by-nc/4.0/), which permits any noncommercial use, distribution, and reproduction in any medium, provided you give appropriate credit to the original author(s) and the source, provide a link to the Creative Commons license, and indicate if changes were made.

\section{REFERENCES}

1. Martin GP, Sperrin M, Bagur R, et al. Pre-implantation balloon aortic valvuloplasty and clinical outcomes following transcatheter aortic valve implantation: a propensity score analysis of the UK Registry. J Am Heart Assoc. 2017;6(2):e004695.

2. Kappetein AP, Head SJ, Genereux P, et al. Updated standardized endpoint definitions for transcatheter aortic valve implantation: the Valve Academic Research Consortium-2 consensus document. Eur Heart J. 2012;33(19):2403-18.

3. Bavry AA, Aalaei-Andabili SH, Karimi A, et al. Comparison of different invasive hemodynamic measurements as a prediction tool for mortality after transcatheter aortic valve replacement in men: a retrospective observational study. Cardiol Ther. 2017;6(2):251-9.

4. Carabello BA, Barry WH, Grossman W. Changes in arterial pressure during left heart pullback in patients with aortic stenosis: a sign of severe aortic stenosis. Am J Cardiol. 1979;44(3):424-7.

5. Ferrera C, Nombela-Franco L, Garcia E, et al. Clinical and hemodynamic results after direct transcatheter aortic valve replacement versus pre-implantation balloon aortic valvuloplasty: a case-matched analysis. Catheter Cardiovasc Interv. 2017;90(5):809-16.

6. Leclercq F, Robert P, Labour J, et al. Prior balloon valvuloplasty versus DIRECT transcatheter Aortic Valve Implantation (DIRECTAVI): study protocol for a randomized controlled trial. Trials. 2017;18(1):303. 